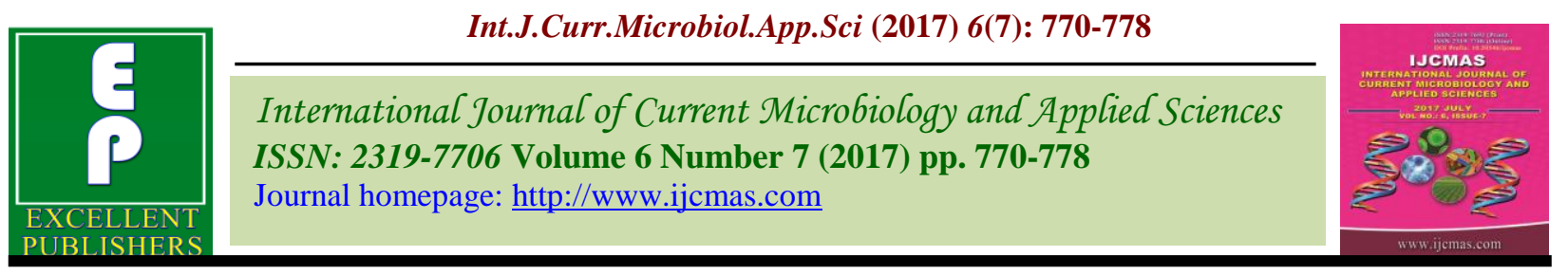

Original Research Article

https://doi.org/10.20546/ijcmas.2017.607.096

\title{
Detection of Methicillin Resistant, ESBL and Amp C Producing Uropathogens from a Tertiary Care Hospital in North India
}

\author{
Shetty Jeevan* and Afroz Zarrin \\ Department of Microbiology, G.S Medical College and Hospital (Chaudhary Charan Singh \\ University), Pilkhuwa, Uttar Pradesh, India \\ *Corresponding author
}

\section{A B S T R A C T}

Uropathogens are responsible for causing one of the commonest infectious diseases in clinical practice. Empirical treatment of this infection is dependent on antibiotic sensitivity pattern of common isolates prevalent in the region. The aim of the study was to determine resistant pattern of urinary isolates and occurrence of extended spectrum beta- lactamase (ESBL) and Amp C production in them. Patients clinically suspected to be having urinary tract infection (UTI) were included in the study. This study was conducted in the Department of Microbiology, G. S Medical College \& Hospital, Pilkhuwa, Uttar Pradesh over a period of 9 months from August 2016 to April 2017. The isolates were identified by conventional microbiological methods and antimicrobial susceptibility testing was done by

\begin{tabular}{|c|}
\hline Keywords \\
\hline $\begin{array}{l}\text { Detection, } \\
\text { Uropathogens, } \\
\text { Tertiary care and } \\
\text { North India. }\end{array}$ \\
\hline Article Info \\
\hline $\begin{array}{l}\text { Accepted: } \\
\text { 14 June } 2017 \\
\text { Available Online: } \\
\text { 10 July } 2017\end{array}$ \\
\hline
\end{tabular}
Kirby- Bauer disk diffusion method. Detection of Extended Spectrum Beta-Lactamase (ESBL) production in Gram negative organisms was done by phenotypic confirmatory disc diffusion test (PCDT) and Methicillin resistance in Staphylococcus was carried out according to Clinical Laboratory Standards Institute (CLSI) guidelines. Amp C betalactamase detection was done by Amp C Disc test. Out of the 210 (16.9\%) uropathogens obtained from 1236 urine samples, $166(13.4 \%)$ were gram negative bacilli and 44(3.5\%) were gram positive cocci. Among Staphylococcus aureus (S. aureus), 24 isolates were methicillin sensitive Staphylococcus aureus (MSSA) and 12 methicillin resistant Staphylococcus aureus (MRSA). Among 166 gram negative bacilli, Escherichia coli (E. coli) was the commonest isolate $(57.8 \%)$ followed by Klebsiella species $(32.5 \%)$. UTI was more common in females $(74.3 \%)$ compared to male $(25.7 \%)$ population. Age group of 20-30 years showed higher incidence and females predominated males in all age groups except among $\geq 60$ years. Among gram negative bacilli, Imipenem showed 100\% sensitivity while in gram positive cocci all isolates were sensitive to Vancomycin. ESBL production was observed in 65(39.1\%) and Amp C production in 41 (24.6\%) isolates of gram negative bacilli. E. coli $(30.1 \%)$ was the predominant ESBL producer while plasmid mediated Amp C beta-lactamase was observed mainly in Klebsiella species (19.3\%) both of which are statistically significant $(\mathrm{p}<0.05)$. This study reveals high prevalence of MRSA, ESBL and Amp C producing urinary pathogens in this region. It is necessary for both clinicians and microbiologists to recognize this high rate of multi drug resistance, so that effective measures may be adopted to control their spread.

\section{Introduction}

Urinary tract infections is one of the common problem diagnosed and treated in urgent care medical practice. UTI's can affect both the lower urinary tract (cystitis) as well as upper urinary tract (pyelonephritis). It is also one of the most common causes of nosocomial 
infection among hospitalized patients. Patients with cystitis usually present with symptoms of dysuria, urinary frequency, hesitancy or urgency, suprapubic discomfort and sometimes even with hematuria. Pyelonephritis typically presents with fever and chills, abdominal pain, nausea, vomiting, malaise and with or without concomitant lower urinary tract signs (Gibson and Toscano, 2012). Urine formed in the kidneys acts as a good culture medium for growth of pathogens and the most commonly incriminated agents are Gram negative bacilli and Staphylococcus species. UTI's are more common in female compared to men because of short urethra and proximity to anus. Sexual intercourse and pregnancy increases their liability to infection. Stasis of urine due to the pressure of gravid uterus adds up to the risk of UTI in pregnant women (Ezeigbo et al., 2016) The common uropathogens are Escherichia coli, Staphylococcus species, Klebsiella pneumoniae, Pseudomonas aeruginosa, Proteus species, Enterococci and Candida albicans. Extensive and inappropriate use of antimicrobial agents has invariably resulted in the development of antibiotic resistance in recent years causing a major problem worldwide (Shah et al., 2015).

ESBL's are plasmid mediated $\beta$-lactamase produced by Gram negative rods which is capable of hydrolyzing penicillin, narrow and broad spectrum cephalosporins and monobactams, thus rendering these antibiotics ineffective in use. Clavulanic acid, sulbactam and tazobactam are generally used as $\beta$ lactamase inhibitors along with the $\beta$-lactam antibiotics to inhibit ESBL enzyme (Devaraju and Rajesh, 2016). Amp C $\beta$-lactamases are cephalosporinases produced by members of the Enterobacteriaceae and other organisms. They mediate resistance to penicillins, first, second and third generation cephalosporins, cephamycins and $\beta$-lactamase inhibitors. Hydrolysis of cephamycins such as cefoxitin is a property that helps to distinguish Amp C's from ESBL's. These enzymes are inducible and overexpression due to mutation is commonly noticed (El-Kazzazand El-Kheir, 2015). There is a paucity of data about the prevalence of plasmid mediated Amp C $\beta$ lactamases ( $\mathrm{p}$ Amp $\mathrm{C}$ ) which are associated with multiple antibiotic resistance.

Among the Gram positive isolates, Staphylococcus aureus is showing increasing resistance to methicillin. Staphylococci and Enterococci appear to become drug resistant readily than most bacteria and constitute serious challenge for medical practice (Raja and John, 2014). Uncomplicated UTI is commonly seen in sexually active women and there is structurally and functional normal urinary tracts. Complicated UTI is usually associated with co-morbid conditions and includes abnormalities of urinary tract or presence of foreign body (Chowdhury and Parial, 2015). Physicians need to be aware of the resistance pattern of uropathogens prevalent locally. There are no studies about the prevalence of ESBL, Amp $\mathrm{C}$ and methicillin resistance of urinary isolates in this region. In this background the study was conducted which helps in the proper treatment of the patients and prevent further development of bacterial drug resistance.

\section{Materials and Methods}

Aprospective observational study was conducted in the Department of Microbiology, G.S Medical College and Hospital, Pilkhuwa, Uttar Pradesh over a period of nine months from August 2016 to April 2017. The study was approved by the ethical committee of the institution. Clean catch midstream urine was collected from each patient in a $30 \mathrm{ml}$ sterile screw capped container. Informed consent was obtained from all patients prior to the collection of sample. The semi-quantitative technique was 
used to calculate significant bacteriuria. Using $0.01 \mathrm{ml}$ calibrated wire loop, samples were inoculated on to 5\% blood agar and MacConkey agar plates. Culture plates were incubated at $37^{\circ} \mathrm{C}$ for $18-24$ hours and colonies are picked up to perform identification (Collee et al., 1996).

A total of 1236 midstream samples were processed for significant bacteriuria and 210 samples yielded the pathogens. After the isolates were identified by conventional microbiological methods, antimicrobial susceptibility testing was done by KirbyBauer disk diffusion method on MuellerHinton agar plates using Amoxyclav $(20 / 10 \mu \mathrm{g})$, vancomycin $(30 \mu \mathrm{g})$, cefazolin $(30$ $\mu \mathrm{g})$, gentamicin $(10 \mu \mathrm{g})$, ofloxacin $(5 \mu \mathrm{g})$, nitrofurantoin $(300 \mu \mathrm{g})$, tetracycline $(30 \mu \mathrm{g})$, amikacin $(30 \mu \mathrm{g})$, cefotaxime $(30 \mu \mathrm{g}), \mathrm{co}-$ trimoxazole $(1.25 / 23.75 \mu \mathrm{g})$, ciprofloxacin $(5 \mu \mathrm{g})$ and imipenem $(10 \mu \mathrm{g})$ (Himedia Lab, Mumbai).

\section{Detection of methicillin resistance in Staphylococcus aureus}

Methicillin resistance in $S$. aureus was detected based on Clinical and Laboratory Standards Institute (CLSI) recommendations using cefoxitin $(30 \mu \mathrm{g})$ disc on a swab inoculated Mueller Hinton Agar (MHA) plate and incubated at a temperature of $35^{\circ} \mathrm{C}$ for 24 hours (CLSI, 2014). S. aureus ATCC 25923 was used for the purpose of quality control (Figure 1).

\section{Detection of Extended Spectrum Beta- Lactamase (ESBL) by phenotypic confirmatory disc diffusion test (PCDT)}

The gram negative bacterial isolates which showed resistance or reduced zone of inhibition to any of the third generation cephalosporins were selected. Disks of Cefotaxime $(30 \mu \mathrm{g})$ and Ceftazidime $(30 \mu \mathrm{g})$ alone and in combination with $\beta$-lactamase inhibitor clavulanic acid $(10 \mu \mathrm{g})$ were used to perform the test. Cefotaxime/Ceftazidime (30 $\mu \mathrm{g})$ and Cefotaxime/ Ceftazidime- clavulanic acid $(30 \mu \mathrm{g} / 10 \mu \mathrm{g})$ were placed on the MHA plate and these plates were incubated overnight at $37^{\circ} \mathrm{C}$. An increase in zone diameter of $\geq 5 \mathrm{~mm}$ with combination disc in comparison to third generation cephalosporin disc alone was considered to be an ESBL producer (Figure 2). Klebsiella pneumoniae ATCC 700603 and E. coli ATCC 25922 were used as ESBL positive and negative controls (CLSI, 2014).

\section{Amp C disc test}

All Gram negative urinary isolates with reduced susceptibility to cefoxitin and resistant to third generation Cephalosporins were tested for Amp C disc test. Amp C disks prepared with sterile paper discs are moistened with $20 \mu \mathrm{l}$ sterile saline and several colonies of test organisms were applied on the disc.

A lawn culture of E. coli ATCC 25922 is prepared on Mueller Hinton Agar plate and 30 $\mu \mathrm{g}$ of cefoxitin discs was placed on it. Now inoculated Amp $\mathrm{C}$ discs are placed almost touching the cefoxitin discs and one negative control was put up. The plates are inverted and incubated overnight at $35^{\circ} \mathrm{C}$ in ambient air (Figure 3). After incubation the plates were examined for indentation or flattening of the zone of inhibition beside Amp $\mathrm{C}$ discs (positive result). The absence of a distortion or no flattening is considered as negative for Amp C production (Black et al., 2005; Kolhapure et al., 2015).

Data were entered and analyzed using SPSS (Statistical Package for Social Science) program version 24 and statistical significance was considered when $\mathrm{p}$ value was less than 0.05 . 


\section{Results and Discussion}

A total of $210(16.9 \%)$ uropathogens were isolated from 1236 urinary samples, out of which 166 (13.4\%) were Gram negative bacilli and $44(3.5 \%)$ were Gram positive cocci. Out of these 210 isolates, $156(74.3 \%)$ were from female and 54from male (25.7\%) and inpatients sample $(56.2 \%)$ was more than outpatients (43.8\%). The highest susceptible age group to UTI was between $20-30$ years (30.5\%) followed by $30-40$ years $(24.7 \%), 40-$ 50 years $(14.3 \%), \geq 60$ years $(14.3 \%), 50-60$ years $(8.6 \%)$ and least in age group $\leq 20$ years $(7.6 \%)$ (Figure 4). Females are more susceptible than males in all age groups except among $\geq 60$ years. Females almost accounted for $96.9 \%$ of UTI in the age group of 20-30 years which is statistically very significant $(\mathrm{p}<0.05)$.

Out of 44 Gram positive cocci, MSSA $(54.5 \%)$ was the predominant isolate followed by MRSA (27.2\%), Enterococcus faecalis (13.6\%) and Staphylococcus saprophyticus (4.5\%). Among Gram negative bacilli, Escherichia coli was the most frequently isolated organism $(57.8 \%)$, followed by Klebsiella species (32.5\%), Acinetobacter species (3.6\%), Pseudomonas aeruginosa (3.6\%) and Proteus mirabilis (2.4\%). K. pneumoniae accounted for two-third isolates among Klebsiella species while $K$. oxytoca formed the remaining one-third.

All Gram positive bacteria obtained from this study were found to be sensitive to vancomycin $(100 \%)$ followed by cefazolin $(59.1 \%)$, gentamicin $(54.5 \%)$, ofloxacin $(50$ $\%)$ amoxyclav (45.5\%), nitrofurantoin (40.9\%), ciprofloxacin (31.8\%), cotrimoxazole $(22.7 \%)$ and tetracycline $(18.2 \%)$ (Figure 5). Gram negative bacilli in this study were found to be $100 \%$ sensitive to imipenem, followed by amikacin (65.1\%), nitrofurantoin $(53 \%)$, gentamicin $(48.2 \%)$, cefotaxime $(31.3 \%)$, ofloxacin $(27.7 \%)$, cotrimoxazole $(25.3 \%)$, tetracycline $(24.1 \%)$, ciprofloxacin $(20.5 \%)$, and amoxyclav (12\%) (Figure 6).

The Clinical microbiological laboratories usually notice variations in in vitro pattern of resistance to $\beta$ - lactam agents among both gram positive and gram negative bacteria. MRSA isolates being resistant to many antibiotic classes have become an important public health concern. ESBL producing uropathogens has been the major mechanism of resistance to beta-lactam agents and often possess resistance determinants to other antibiotic groups such as fluroquinolones and aminoglycosides. Plasmid mediated Amp C are of increasing concern as attempt to detect them are not reported and typically these enzymes are associated with multiple antibiotic resistances. E. coli (30.1\%) was the predominant ESBL producer (50/166) among Gram negative bacilli while plasmid mediated Amp C beta-lactamase was observed predominantly in Klebsiella species (19.3\%) both of which are statistically significant $(\mathrm{p}<0.05)$ (Table 1). ESBL production was observed in nine isolates of Klebsiella and in two isolates each of Pseudomonas aeruginosa, Acinetobacter species and Proteus mirabilis. AmpC production was also seen in isolates of E. coli (4), P. aeruginosa (2) and Acinetobacter spp. (3). Co-expression of ESBL and Amp C was significantly observed in ten isolates of Klebsiella spp.

Uropathogens were predominantly isolated in females $(74.3 \%)$ than men as seen in several other studies. UTI's are more seen in female than men because of their shorter urethra and their close proximity to anus. The highest susceptible age group to UTI in this study was between $20-30$ years $(30.5 \%)$ followed by 30 40 years $(24.7 \%)$ and least in the age group $\leq$ 20 years $(7.6 \%)$. Females predominated males in all age groups except among $\geq 60$ years. 
Incidence of UTI in men increases as age advances probably because of hypertrophy of prostate and other related problems of old age (Razak and Gurushantappa, 2012).

S. aureus causing UTI is higher in our study (17.14\%) compared to the several reports from other regions where Enterococcus faecalis was the predominant isolate among Gram positive cocci (Das and Banerjee, 2015;
Mishra et al., 2016).One study has found Enterococcus species comparatively lower than Staphylococcus species which is similar to this study (Tuli et al., 2016).

MRSA constituted one third of S. aureus isolates in this study and rarely its role in UTI has been reported (Venkatesh et al., 2016).All isolates of S. aureus showed $100 \%$ sensitivity to Vancomycin.

Fig.1 MSSA and MRSA identified by using cefoxitin disc (sensitive/resistant)

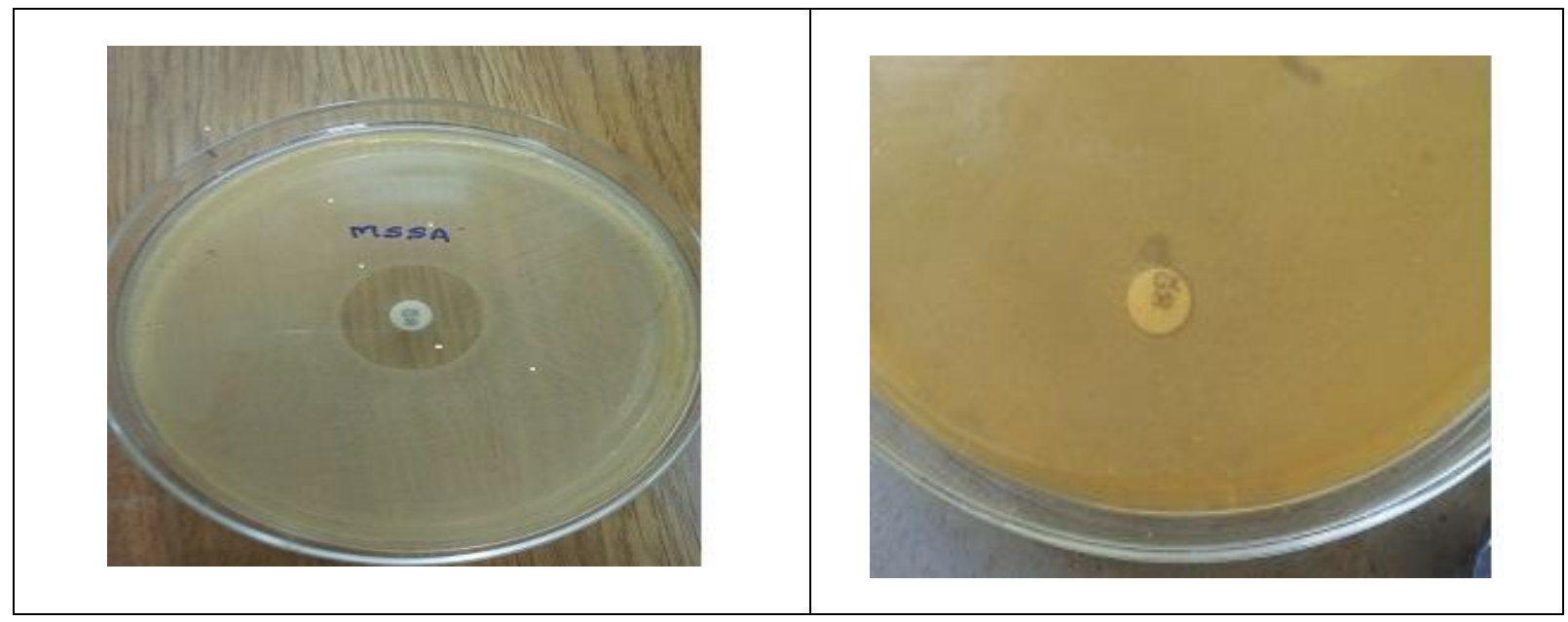

Fig.2 ESBL detection by using Cefotaxime (Ctx) and Cefotaxime/ Clavulanic acid (CeC) Discs

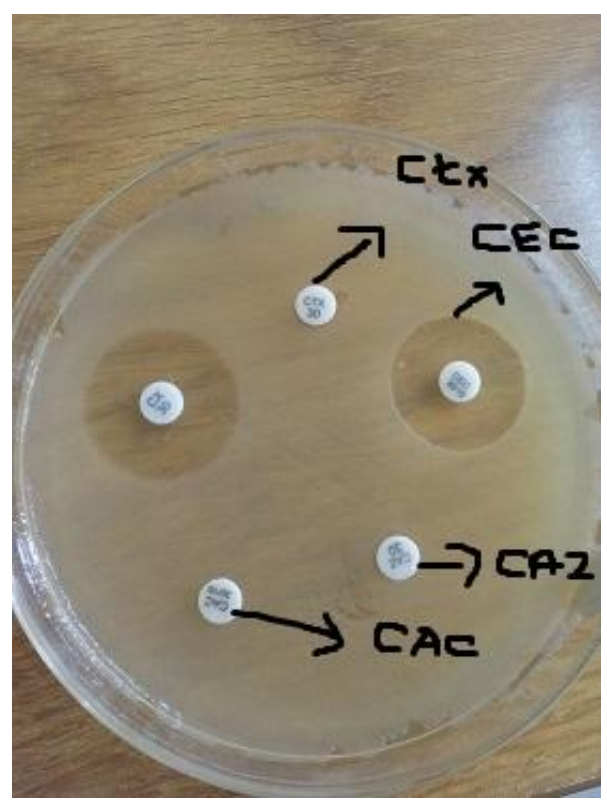


Fig.3 Amp C detection using Cefoxitin (Cx) and adjacent Amp C disc ( 2 test organism above showing distorted zone and negative control at the bottom)

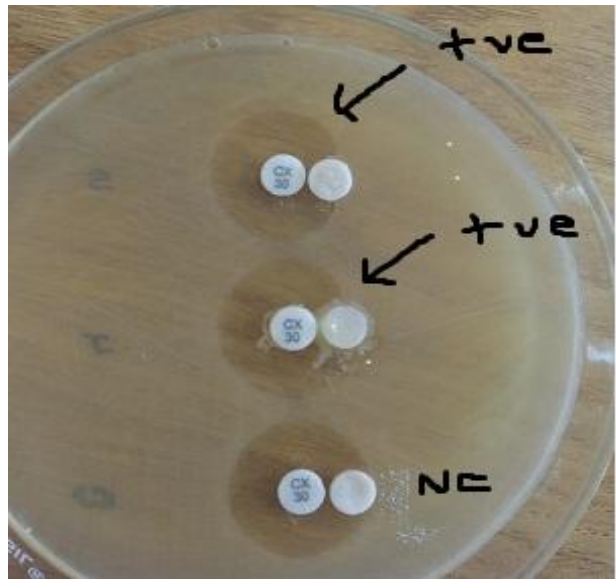

Fig.4 Age-wise distribution of positive cultures yielding uropathogens

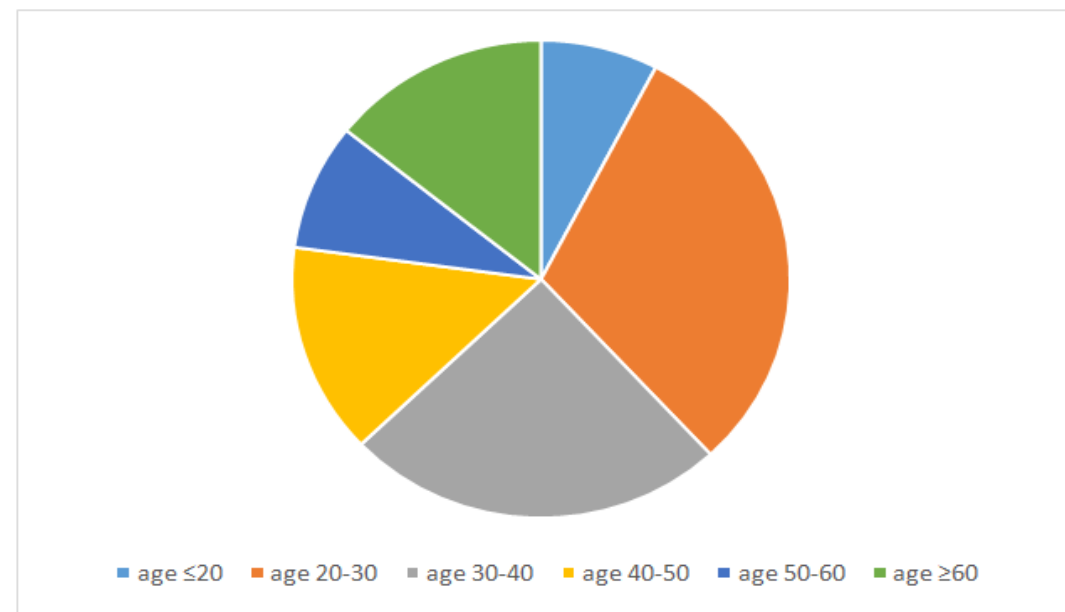

Fig.5 Antibiotic susceptibility pattern of Gram positive uropathogens

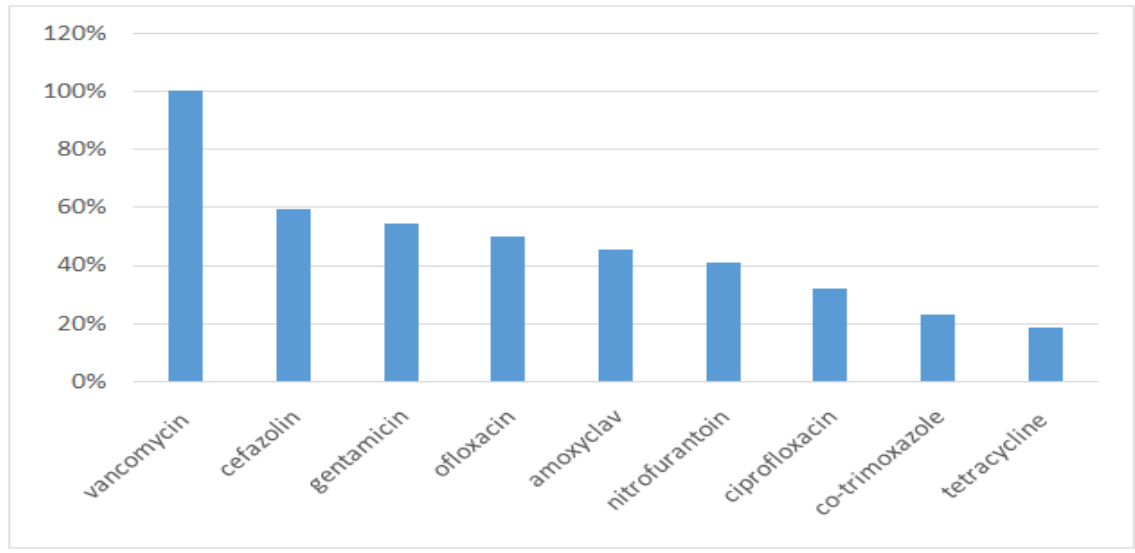


Fig.6 Antibiotic susceptibility pattern of Gram negative uropathogens

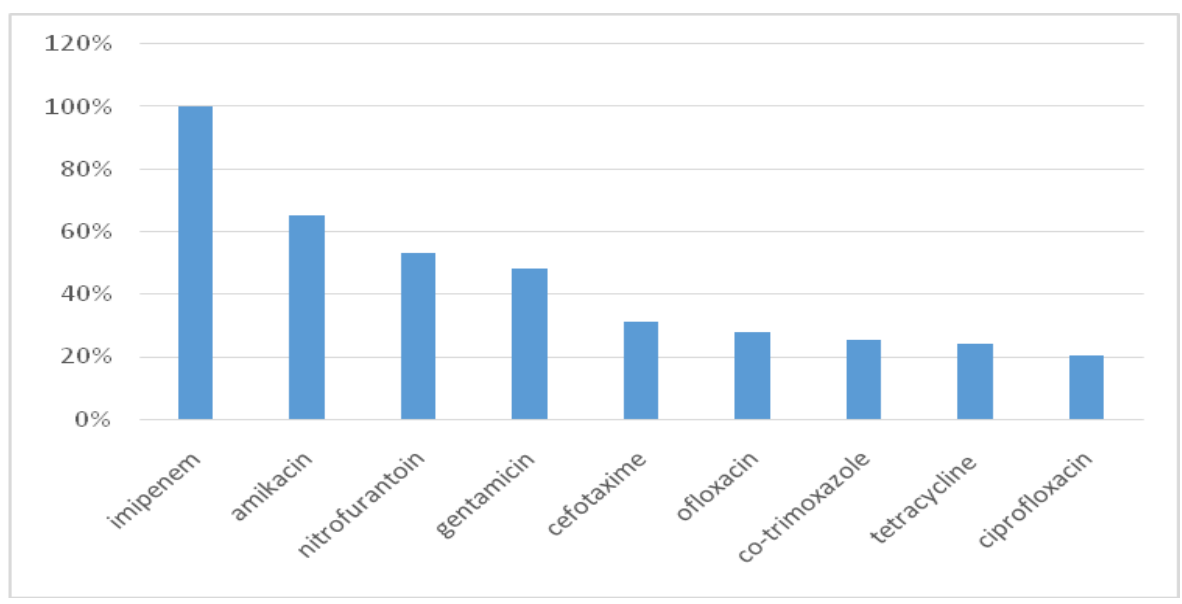

Table.1 Prevalence of ESBL and Amp C producing Gram negative uropathogens

\begin{tabular}{|l|l|l|l|l|}
\hline Gram negative bacilli & $\begin{array}{l}\text { Number of } \\
\text { isolates } \\
\text { screened }\end{array}$ & $\begin{array}{l}\text { ESBL } \\
\text { positive }\end{array}$ & $\begin{array}{l}\text { Amp C } \\
\text { positive }\end{array}$ & $\begin{array}{l}\text { ESBL + } \\
\text { Amp C }\end{array}$ \\
\hline Escherichia coli & 96 & 50 & 4 & 2 \\
\hline Klebsiella species & 54 & 9 & 32 & 10 \\
\hline Pseudomonas aeruginosa & 6 & 2 & 2 & 0 \\
\hline Acinetobacter species & 6 & 2 & 3 & 1 \\
\hline Proteusmirabilis & 4 & 2 & 0 & 0 \\
\hline Total & 166 & 65 & 41 & 13 \\
\hline
\end{tabular}

Escherichia coli (57.8\%) was the most frequently isolated organism among gram negative bacilli, followed by Klebsiella species (32.5\%), Acinetobacter species (3.6\%), Pseudomonas aeruginosa (3.6\%) and Proteus mirabilis (2.4\%). This is in consistent with several studies where the predominant pathogen was E. coli followed by Klebsiella (Razak and Gurushantappa, 2012; Tuli et al., 2016).

Gram negative bacilli in this study were found to be $100 \%$ sensitive to imipenem, followed by amikacin (65.1\%), nitrofurantoin (53\%), gentamicin $(48.2 \%)$, cefotaxime $(31.3 \%)$ and ofloxacin (27.7\%). High resistance of uropathogens to different antibiotic classes has been reported in another study in the surrounding region (Rangari et al., 2015). But there is lack of information regarding ESBL and Amp C production among these isolates which is of clinical importance and this study is useful in this regard.

This study observed that E. coli (30.1\%) was the predominant ESBL producer among Gram negative bacilli while plasmid mediated Amp C beta-lactamase was observed predominantly in Klebsiella species (19.3\%). This significant beta-lactamase production among E. coli and Klebsiella species is of grave concern as they are invariably associated with resistance to multiple antibiotics and therapeutic failure if they are not detected routinely in the laboratories.

Several studies across the globe have reported high incidences of ESBL and Amp C in their set up. Study done in Iran observed $62.7 \%$ and $5 \%$ of $E$. coli were ESBL and Amp C producers respectively (Shayan and Bokaeian, 2015). 
High prevalence of ESBL (32.8\%) and AmpC $(41.2 \%)$ producing bacilli belonging to Enterobacteriaceae were detected in another study (Madhavan and Jayalakshmi, 2016). Similar high prevalence of ESBL and AmpC beta-lactamase from urinary isolates has been observed in other studies. ESBL production was observed in $66.1 \%$ and Amp C production in $46.1 \%$ of the urinary isolates (Kaur et al., 2016). A total of $72.41 \%$ isolates were ESBL positive and $21.76 \%$ AmpC producers from another study which included urinary isolates (Shinu et al., 2014).

ESBL production in E. coli and Klebsiella spp. together constituted $35.5 \%(59 / 166)$ in this study. ESBL producers in E. coli and Klebsiella species among urinary isolates in another centre were $57.79 \%$ (Kulkarni et al., 2016). Klebsiella species was the predominant producer of AmpC beta-lactamse $(19.3 \%)$ and was observed in $E$. coli $(2.4 \%), \quad P$. aeruginosa (1.2\%) and Acinetobacter spp (1.8\%) in this study. E. coli with both ESBL and AmpC producers have been noted in many studies. Forty-eight percent of E. coli were ESBL producers and coexistence of ESBL and AmpC was observed in $3.17 \%$ of $E$. coli infection in HIV patients (Buzayan and El-Garbulli, 2014). ESBL production was observed in $52.6 \%$ and AmpC production in $8 \%$ of E. coli from UTI and interestingly invariably all isolates of AmpC producers were also found to be ESBL positive (Gupta et al., 2013). The resistance mechanism observed in some of these gram negative bacilli in this study may be due to different mechanism such as mutation in porins or due to efflux pumps. Molecular studies using polymerase chain reaction (PCR) would be of great help in studying the involvement of different genes of beta-lactamase and their expression in these uropathogens.

Because of the rapid change in antibiotic susceptibility pattern of uropathogens over time, studies should be regularly done to understand the mechanism of resistance in these isolates. In resource poor settings where facilities of PCR is not available or not routinely done, phenotypic methods of detection of ESBL by PCDT and AmpC detection by AmpC Disc test is invariably the most economical and reliable test. Prevalence of MRSA (27.2\%) among gram positive cocci and ESBL (39.1\%) and AmpC (24.6\%) production among gram negative bacilli in this region is a worrisome factor.

\section{References}

Black JA, Moland ES, Thomson KS. 2005. Amp $\mathrm{C}$ disk test for detection of plasmid mediated Amp C $\beta$ - lactamases in Enterobacteriaceae lacking chromosomal Amp C beta-lactamases. J Clin. Microbiol. 43(7):3110-3113.

Buzayan MM, El-Garbulli FR. 2014. Detection of ESBL and AmpC $\beta$-lactamases producing in uropathogen Escherichia coli isolates at Benghazi Center of Infectious Diseases and Immunity. Int J CurrMicrobiol App Sci., 3(2):145-153.

Chowdhury S, Parial R. 2015. Antibiotic susceptibility patterns of bacteria among Urinary tract infection patients in Chittagong, Bangladesh. SMU Med. J., 2(1):114-125.

Clinical and Laboratory Standards Institute. 2014. Performance standards for antimicrobial susceptibility testing; Twenty fourth informational supplement, M100-S24. Wayne, PA

Collee JG, Fraser AG, Marmion BP, Simmons A. 1996. Mackie and McCartney Practical Medical Microbiology. $14^{\text {th }}$ Ed. New York: Churchill Livingstone.

Das A, Banerjee T. 2015. Prevalence of Urinary tract infections and susceptibility pattern of uropathogens in women of reproductive age group from North India. J Advances Med. 4(1-2):05-09

Devaraju AD, Ramachander Rajesh. 2016. Occurrence of various $\beta$-lactamase enzyme producing Enterobacteriaceae in the hospital effluent: a wake-up call. Int J Med Sci Public Health, 5(6):1204-1208.

El-Kazzaz SS, Abou El-Kheir NT. 2015. Amp C and metallo beta-lactamases producing Gram negative bacteria in patients with hematological malignancy. Afr J Microbiol 
Res., 9(18):1247-1254.

Ezeigbo OR, Nnadozie RIA, Asuoha-Chuks N, Ojiako VU, Awurum IN, Ugochukwu MG. 2016. Incidence of Urinary Tract Infection (UTI) among pregnant women attending antenatal clinics at some selected Hospitals in Aba, Southeastern Nigeria. Int J Curr. Microbiol App Sci. 5(1):193-219.

Gibson K, Toscano J. 2012. Urinary Tract Infection Update. American J Clin. Med., 9(2):82-86.

Gupta V, Rani H, Singla N, Kaistha N, Chander J. 2013. Determination of Extended Spectrum $\beta$-lactamases and AmpC production in Uropathogenic isolates of Escherichia coli and Susceptibility to Fosfomycin. J Lab Physicians, 5(2):90-93.

Kaur J, Mahajan G, Chand K, Sheevani, Chopra S. 2016. Enhancing phenotypic detection of ESBL in AmpC co-producers by using cefepime and tazobactam. J ClinDiag Res., 10(1):05-08.

Kolhapure RM, Kumar A, Rajkumar HRV. 2015. Coexpression of ESBL, Amp C and MBL in gram negative bacilli. Int J Res Med Sci., 3(10):2698-2703.

Kulkarni DM, Bardapurkar SA, Nilekar SL, More SR. 2016. Prevalence of extended spectrum $\beta$-lactamase (ESBL) producing E. coli and Klebsiella species in urinary isolates. IOSR J Dent Med Sci., 15(6):26-29

Madhavan A, Jayalakshmi V. 2016. Occurrence of extended spectrum beta lactamase, AmpC and MBLase producers among multidrug-resistant Enterobacteriaceae causing urinary tract infection in a tertiary health care teaching hospital. J Acad Clin Microbiol, 18(2):80-85

Mishra R, Jayesh, Singh AK, Jasuja K. 2016. Bacteriological profile and sensitivity pattern of microrganisms causing Urinary tract infection at a tertiary care center in eastern Uttar Pradesh. Int J Biomed
AdvRes.7 (6):292-297.

Raja MM, John SA. 2014. Multidrug resistance profile of urinary tract infected Gram positive pathogenic bacterial isolates. Int $J$ Infect., 2(1):e22774.

Rangari AA, Sharma S, Tyagi N, Singh P, Singh G, Thakur R. 2015. Antibiotic susceptibility pattern of bacterial uropathogens isolated from patients at a tertiary care hospital in Western Uttar Pradesh of India. Int J Curr Microbiol App Sci., 4(10):646-657.

Razak SK, Gurushantappa V. 2012. Bacteriology of urinary tract infection and Antibiotic susceptibility pattern in a tertiary care hospital in South India. Int J Med Sci Pub Health. 1(2):109-112.

Shah LJ, Vaghela GM, Mahida H. 2015. Urinary tract infection: Bacteriological profile and its antibiotic susceptibility in Western India. Nat J Med Res., 5(1):71-74.

Shayan S, Bokaeian M. 2015. Detection of ESBL and AmpC producing E. coli isolates from urinary tract infections. Adv Biomed Res., 4:220-223.

Shinu P, Bareja R, Goyal M, Singh VA, Mehrishi P, Bansal M etal. 2014. Extended-spectrum $\beta$-lactamase and AmpC $\beta$-lactamase production among Gram-negative bacilli isolates obtained from Urinary tract infections and Wound infections. Ind J Clin Practice, 24(11):1019-1026.

Tuli L, Rai S, Dilshad A, Singh DK. 2016. Bacteriological profile and antimicrobial susceptibility pattern of isolates from Urinary tract infections in eastern Uttar Pradesh, India. Int J Microbiol App Sci., 5(3):428-435.

Venkatesh RK, Prabhu MM, Nandakumar K, Pai KSR. 2016. Urinary tract infection treatment pattern of elderly patients in a tertiary hospital setup in South India: A Prospective study. J Young Pharmacists, 8(2):108-113.

\section{How to cite this article:}

Shetty Jeevan and Afroz Zarrin. 2017. Detection of Methicillin Resistant, ESBL and Amp C Producing Uropathogens from a Tertiary Care Hospital in North India. Int.J.Curr.Microbiol.App.Sci. 6(7): 770778. doi: https://doi.org/10.20546/ijcmas.2017.607.096 